\title{
Is the prevalence of dementia changing?
}

\section{“...more recently born older individuals are, on average, in better physical health and appear to develop dementia later..."}

It is important that we know how many individuals have dementia. This is for reasons such as being able to provide appropriate care and services, and also so that we can estimate future numbers with as much accuracy as possible. It is well known that as the proportion of older individuals in the population continues to increase, so too will the prevalence of dementia, as age is by far the biggest risk factor for the condition. This phenomenon is worldwide as has been demonstrated by a series of epidemiological studies and also by the report by Alzheimer's Disease International [101]. Quoted figures for the number of prevalent cases have gradually increased over the last couple of decades. In the UK, the figure currently used in policy is approximately 800,000 individuals [102], and globally approximately 36 million individuals [101].

However, in recent decades there have been other changes. In higher income countries, more recent birth cohorts of older individuals have enjoyed better health, education and nutrition than their predecessors, and numbers of deaths from vascular disease have fallen. In general, longevity has increased and there may be more centenarians alive now than have existed in the history of the world. Pension and retirement ages are also creeping upwards. At an individual level, a fit, healthy person of 65 years of age will bristle with resentment at the suggestion that they are 'old'.

Consequently, it is of great importance to know whether these cohort changes have affected the epidemiology of dementia. There has long been some circumstantial evidence that individuals are tending to develop dementia at older ages than was previously the case. This is reflected in the increased average ages of patients referred to memory assessment services over the last couple of decades. Not only that, but patients are referred for assessment at earlier stages of disease progression than used to be the case, so the increased age is not due to dementia being overlooked or neglected. A second strand of evidence was that early epidemiological studies seemed to report higher prevalences than more recent ones, although it was possible that these differences were due to methodological factors rather than genuine difference in disease rates [1]. The only way to address this problem adequately is to perform a repeat study after a suitable period of time, and evidence is now beginning to accumulate.

Several studies in the last decade have suggested that the prevalence and/or the incidence of dementia may be decreasing. For example, Manton et al. examined US Medicare data at five time points between 1982 and 1999 and reported a decrease in prevalence of severe cognitive impairment among individuals $>65$-yearold individuals from 5.7 to $2.9 \%$ during this period [2]. Also in the USA, Langa et al. found that $12.2 \%$ of individuals, studied in 1993 , aged over 70 years of age had cognitive impairment compared with $8.7 \%$ in 2002 [3]. However, in those who did develop moderate or severe cognitive impairment, mortality rates increased over time, consistent with the compression of morbidity hypothesis - in that, more recent cohorts appear to be healthier and have more cognitive reserve but decompensate faster once dementia does occur. Lobo et al. found that dementia prevalence in individuals over 65 years of age fell between two waves of study in Zaragoza (Spain), from 5.2 to $3.9 \%$, but that this decrease was only statistically significant in men [4]. In the Rotterdam study, there was a decrease in the age-adjusted incidence of dementia between cohorts recruited in 1990 and 2000, with decreased mortality rates and evidence on brain imaging of larger brain volumes (less atrophy) and less cerebral vascular disease [5]. Qiu et al. compared two epidemiological studies of individuals over the age of 75 in Stockholm, from 1987 to 1989 and 2001 to 2004 [6]. They found that the prevalence of dementia remained stable over this time but the mean survival of individuals with dementia increased, suggesting that the incidence of dementia (number of new cases) may have decreased.

Two recent papers in the Lancet also reported such findings. The first study, Matthews et al., comes from the UK Medical Research Council-funded CFAS [7]. The first wave of CFAS (CFAS I) was conducted between 1989 and 1994, with participants aged 65 years or more, so born at least before 1929. Six centers in England
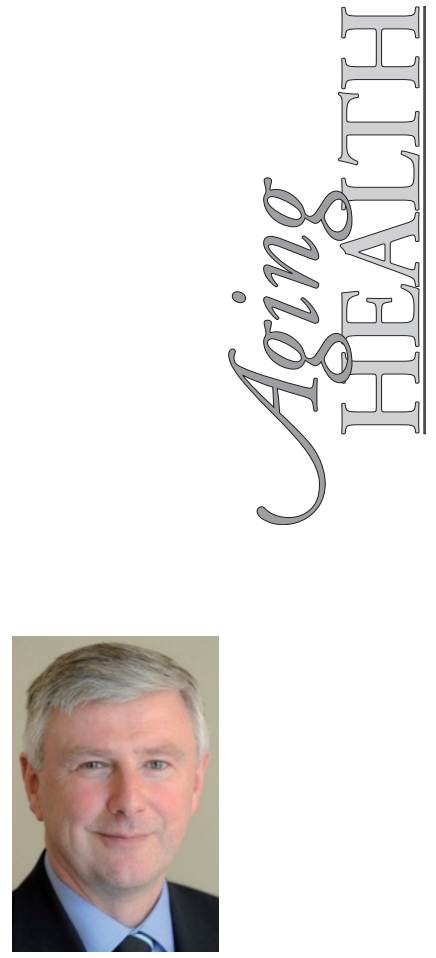

Tom Dening

Institute of Mental Health, University of Nottingham Innovation Park,

Triumph Road, Nottingham, NG7 2TU, UK

*Author for correspondence:

Tel.: +441158230421

tom.dening@nottingham.ac.uk

\section{Keywords}

- cognitive impairment

- dementia • epidemiology

- older people • prevalence

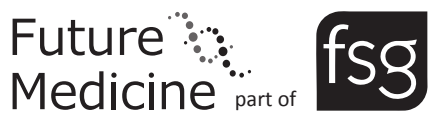


and Wales participated, giving a representative sample. The main findings around prevalence were reported in 1998 (Medical Research Council CFAS, 1998) [8]. The second study (CFAS II) was performed in three of the same areas within England, with the interviews taking place between 2008 and 2011 [8]. Therefore, there was a gap of 18 years and participants in CFAS II could have been born as late as 1946 . The methods of the two studies were identical except CFAS I had a two-stage interview design, which was reduced to one-stage for CFAS II. As there were only three centers in CFAS II, only data from these centers in CFAS I were included in the analyses, to ensure comparability of locations.

The main finding, based on CFAS I results, was that in 1991 there would have been 664,000 individuals with dementia in the UK. Based on this estimate, and projecting forwards on the basis of population aging, there would have been predicted to be 884,000 individuals by 2011 . However, CFAS II data change that estimate to the lower figure of 670,000, in other words 214,000 (24\%) fewer than would have been expected if there had been no change in dementia prevalence in the population.

\section{"Several studies in the last decade have suggested that the prevalence and/or the incidence of dementia may be decreasing."}

This is exciting news as it resonates with the clinical and other impressions that have been described above. It may also go some way to explaining why dementia diagnosis rates in England appear to be unacceptably low - that is, the undiagnosed cases are simply not there to be detected. The figure will also lead us to revise our predictions of future numbers of individuals with dementia, which have been set as high as 1.7 million individuals by 2051 [103].

However, it is important to recognize some limitations and caveats. First of all, we are talking about prevalence rates of dementia, the proportion of people of a given age who have a given condition at any one point in time. The prevalence rate may have fallen but, as the population is continuing to age, the absolute number of cases has not decreased: indeed, there is a small increase in numbers from CFAS I to CFAS II. Although, we can be fairly confident that prevalence rates have fallen because of increased longevity (better health and other population factors), we cannot be assured that they will continue to do so. For example, the impacts of the current epidemic of obesity and it associated morbidity (diabetes, hyperlipidemia and so forth) are yet to work their way into the older population. Additionally, the CFAS studies have some methodological limitations, the most obvious one being the different response rates between CFAS I (80\%) and CFAS II (56\%). This reflects a general change over time in response rates to population based studies, with increased refusals for various reasons. The authors performed sensitivity analyses to adjust for possible biases, but it remains hard to dispel the notion that the different responses in the two studies may have biased the results, possibly in the direction of finding less dementia.

\section{“...there are fewer cases of dementia currently than were predicted."}

The second study also compared two elderly cohorts. Christensen et al. studied two groups of Danish individuals over 90 years of age; the first cohort were born in 1905 and assessed at age 93, and the second cohort were born in 1915 and assessed at age 95 years [9]. Despite being 2 years older at the time of examination, the 1915 group scored significantly higher on the Mini Mental State Examination (mean score of 22.8 vs 21.4 ) and $23 \%$ of the 1915 birth group scored in the normal range of 28-30 compared with $13 \%$ in 1905 group. The earlier sample was larger ( $\mathrm{n}=2262$ vs 1584$)$ but the response rates were similar at $63 \%$, so that does not seem to have been a bias. Given the sample sizes, these differences were highly statistically significant. Although, this is not a study of dementia prevalence as such, it does provide important evidence of improved cognitive function in more recent birth cohorts.

In conclusion, we now have some robust evidence to support our clinical impressions over the last decade or two. Although population aging has continued, more recently born older individuals are, on average, in better physical health and appear to develop dementia later, so that current estimates of dementia prevalence are lower than they were, and there are fewer cases of dementia currently than were predicted. Although this is good news, it should be viewed with some caution as the predicted future numbers of dementia worldwide are already large and still going to grow considerably. Dementia will still be a huge challenge for health and social care systems globally. 
Financial \& competing interests disclosure

$T$ Dening has recently been invited to join the Medical Research Council CFAS management committee but has not received any funding related to this study. The author has no other relevant affiliations or financial involvement

with any organization or entity with a financial interest in or financial conflict with the subject matter or materials discussed in the manuscript apart from those disclosed.

No writing assistance was utilized in the production of this manuscript.

Is dementia incidence declining? Trends in dementia incidence since 1990 in the Rotterdam Study. Neurology 78, 1456-1463 (2012).

6. Qiu C, von Strauss E, Bäckman L, Winblad $\mathrm{B}$, Fratiglioni L. Twenty-year changes in dementia occurrence suggest decreasing incidence in central Stockholm, Sweden. Neurology 80, 1888-1894 (2013).

7. Matthews FE, Arthur A, Barnes LE et al:; on behalf of the Medical Research Council Cognitive Function and Ageing Collaboration. A two-decade comparison of prevalence of dementia in individuals aged 65 years and older from three geographical areas of England: results of the Cognitive Function and Ageing Study I and II. Lancet doi:10.1016/S0140-6736(13)61570-6 (2013) (Epub ahead of print).

8. Cognitive function and dementia in six areas of England and Wales: the distribution of MMSE and prevalence of GMS organicity level in the MRC CFA Study. Psychol. Med. 28, 319-335 (1998).
9. Christensen K, Thinggard M, Oksuzyan A et al. Physical and cognitive functioning of people older than 90 years: a comparison of two Danish cohorts born 10 years apart. Lancet doi:10.1016/S0140-6736(13)60777-1 (2013) (Epub ahead of print).

\section{Websites}

101. Alzheimer's Disease International. World Alzheimer Report 2009. www.alz.co.uk/research/files/ WorldAlzheimerReport.pdf (Accessed 5 August 2013)

102. Alzheimer's Society. Alzheimer's Society Dementia 2012 Report. www.alzheimers.org.uk/site/scripts/ download_info.php?fileID=1389 (Accessed 5 August 2013)

103. Alzheimer's Society. Dementia UK 2007. www.alzheimers.org.uk/site/scripts/ download_info.php?fileID $=2$ (Accessed 5 August 2013) 